\title{
6. ANÁLISIS CUALITATIVO
}

1. Organización de la atención a la salud mental.

Desde la Ley General de Sanidad (1986) hasta la culminación de las transferencias sanitarias del Estado a las CCAA (2002), cada una de ellas ha acometido el proceso de transformación de la asistencia a la salud mental desde un modelo hospitalocéntrico, al modelo comunitario conforme a su situación previa y a su política sanitaria. Se han aprobado Leyes, Decretos y órdenes para la implementación del nuevo modelo de atención a la salud mental.

La plena integración de la atención a la salud mental en la salud general es ya un hecho(tabla 5, item 2). En la actualidad, todas las CCAA tienen un Plan de Salud Mental aprobado (tabla 2, item 1), aunque en muchas de ellas, estos planes han consistido, sobre todo, en un guión de propositos más que un nuevo modelo de planificar y gestionar.

Una verdadera organización de la atención a la salud mental implica un servicio de planificación, gestión y una financiación específica. Sin embargo, observamos la ausencia, en muchas CCAA, de un servicio de Planificación para la salud mental (Cantabria, Melilla, Rioja, Valencia), en la falta de financiación específica (Andalucía, Aragón, Baleares, Canarias, Cantabria, Extremadura, Madrid, Melilla, Navarra, Rioja y Valencia) y en la carencia de una gestión específica (Andalucía, Baleares, Cantabria, Extremadura, Melilla, Rioja y Valencia).

Además, hay que señalar que en muchas CCAA, la gestión (tabla5) no sólo es responsabilidad de la Consejería de Sanidad. En varias de ellas, comparten esta gestión Diputaciones y Consejerías de Servicios Sociales/Conselleria de Bienestar Social (Castilla la Mancha, Extremadura, Valencia y Madrid). Además, y, sobre todo en rehabilitación, esta prestación se realiza a través de convenios con empresas privadas y/ o sin ánimo de lucro, Asociaciones, ONGs.

Siguen existiendo dificultades para una buena coordinación entre administraciones (Canarias, Castilla León y Valencia). También es significativa la falta de coordinación (tabla 6) entre los distintos dispositivos (Aragón, Baleares, Cantabria y Valencia). Solamente existe una Unidad Funcional de coordinación definida en Castilla la Mancha (Comisión Consultiva), Galicia (Servicio de Salud Mental) y Madrid (Oficina Regional de Coordinación de Salud Mental). 
Otro punto importante de la organización de la atención a la salud mental son los sistemas de información y recogida de datos (tabla 7). En España se han realizado pocos estudios epidemiológicos fiables sobre las necesidades de atención en salud mental a la población general. Las estimaciones de dichas necesidades se han realizado, sobre todo, partiendo de la utilización de los dispositivos existentes. Así, vemos que existen pocas CCAA que tengan implantados un sistema de recogida de datos específicos de Salud Mental. Sólo en algunas está el Registro Acumulativo de Casos Psiquiátricos (RACP) y el conjunto mínimo básico de datos (CMBD) y en otras los sistemas de información y de recogidas de datos son los utilizados de forma general por la atención especializada de los hospitales. En la mayoría de los casos la información de los distintos sistema no se gestiona de forma integrada. El nivel de desarrollo de sistemas de recogida de datos y de información que sirvan, no solo como datos epidemiológicos, sino como herramientas de gestión, es insuficiente.

\section{La Red de dispositivos de salud mental en un Área Sanitaria.}

- Unidades/Centros de Salud Mental

. Unidades/Centros de Salud Mental de adultos:

En todas las CCAA existen ya Unidades o Centros de salud mental comunitarios. Pero hemos de señalar que todavía existen Neuropsiquiatras de zona en Madrid, Galicia y Valencia.

Observamos que se da una gran disparidad respecto a la población de referencia para cada uno de las Unidades/Centros de Salud Mental (tabla 9).

En todos las Unidades/Centros el equipo es multidisciplinar contando con Psiquiatras, Psicólogos, Enfermeros, Trabajadores Sociales, Auxiliares de Clínica y, en algunos casos, Terapeutas 0cupacionales. Sin embargo, el número total de profesionales por 100.000 habitantes es totalmente dispar de una CCAA a otra (tabla 12).La actividad asistencial (Primeras, Sucesivas y Total consultas) también es muy desigual de una Comunidad Autónoma a otra (tabla 10).

Lo mismo sucede respecto a los Programas asistenciales definidos en las Unidades/Centros. El Programa básico de coordinación con Atención primaria de Salud no se realiza en Castilla León, Murcia, ni Valencia.

En las CCAA de Asturias, Castilla la Mancha, Castilla León, Euskadi, Navarra y la Rioja, la red de atención a la salud mental y la red de drogodependencias están integradas y las Unidades / Centros tienen el programa de atención a ambas patologías. En Murcia, a pesar de que son redes paralelas, los Centros de salud mental atienden también drogodependencias.

Según las recomendaciones del Cuaderno Técnico de la AEN: “Hacia una atención comunitaria de salud mental de calidad", la estimación de necesidades de profesionales totales en los Centros de Salud Mental se sitúa entre 28 y 32,5 (sin contar con el Programa de Niños y Adolescentes) (7, pág. 59). Esto significa que prevalece, en casi todas las CCAA, una carencia manifiesta respecto al número de profesionales en este primer nivel de atención. 
. Unidades de Salud Mental Infanto-Juvenil

En la Ley General de Sanidad, la atención a la salud mental de la población Infanto-Juvenil se planta como un Programa particular, junto a Psicogeriatría. Sin embargo, varias CCAA han elaborado un Plan de Salud Mental específico para esta población ( Andalucía, Murcia y Castilla la Mancha; en ésta última incluido en el general).

Para la evaluación del dispositivo del primer nivel de atención comunitaria dirigido a la población Infanto-juvenil, introducimos los parámetros específicos de rango de edad y nivel de atención.

El resultado de las respuestas en el cuestionario manifiesta la escasez de recursos tanto estructurales como de profesionales en todas las CCAA. Respecto al número de profesionales necesarios para una población de 100.000 habitantes, el Cuaderno Técnico de la AEN (7, Pág. 59) expresa que, como mínimo son necesarios un total de 4 profesionales $y$, en la actualidad, la media de todas las CCAA (excepto Canarias, Cantabria y Cataluña) se sitúa en un 1,75 .

La denominación para este primer nivel de atención casi siempre es Equipo de Salud Mental Infanto-Juvenil. Sólo Madrid y Navarra lo explicita como "Programa". Existe mucha diversidad respecto al rango de edad de la población atendida que va desde los 0 hasta los 14, 15, 16, 17 y 18 años. Tampoco existe una afinidad respecto al nivel de atención, en unas CCAA pueden derivar directamente desde Atención Primaria de salud y en otras desde las Unidades/Centros de adultos. En Andalucía, las Unidades son también Hospital de Día. El sistema de registro e información también es escaso por lo que no tenemos datos de la actividad asistencial (primeras, sucesivas y total consultas) en seis CCAA (Asturias, Canarias, Cantabria, Castilla León, Cataluña y Extremadura). En casi todas las (CAA se aplican Programas básicos asistenciales y de coordinación.

- Hospitalización

. Hospitalización Breve de Adultos

Según recomienda la Ley General de Sanidad, ya en todas las CCAA, se han creado ya unidades / servicios de hospitalización en los hospitales generales. Sin embargo siguen existiendo Unidades de Hospitalización Breve en los Hospitales Psiquiátricos (Baleares, Cataluña, Euskadi Extremadura, Madrid y Valencia) .

En el Cuaderno Técnico de la AEN (7, Pág. 59) la estimación de necesidades de plazas de hospitalización breve para la población adulta es de entre un 12 y un $15 \mathrm{x}$ 100.000 habitantes. Actualmente, la media en las CCAA se sitúa en torno al 9,95.

Es significativo que en casi todas las CCAA existan Unidades Específicas para Trastornos de la Conducta Alimentaria.

El número total de profesionales $\times 100.000$ habitantes es muy dispar de unas CCAA a otras, desde 5,4 en Navarra hasta 22,21en Melilla. 
. Hospitalización Breve Infanto-Juvenil:

Sólo existe este recurso en las CCAA de Andalucía, Cataluña, Euskadi, Madrid y Valencia, por lo que no puede realizarse una análisis comparativo, dato ya significativo en sí mismo.

Hemos de reseñar, que en varias CCAA ( Andalucía y Castilla León), en el Servicio de Pediatría hay camas asignadas para la población Infanto-Juvenil con trastornos de Salud Mental. Solamente Cataluña cuenta con una Unidad de Crisis para adolescentes.

- Hospitalización Parcial

. Hospitalización Parcial (de Día) de Adultos.

Respecto a este dispositivo hemos de destacar varios puntos:

- Algunos van dirigidos al tratamiento de una patología específica (Navarra y Cantabria).

- No realizan Tratamientos psicoterapéuticos (Castilla León)

- Muchos de ellos no dependen de las administraciones públicas, siendo centros privados concertados (Cantabria, Castilla León, Euskadi y Navarra).

. Hospitalización Parcial (Día) Infanto-Juvenil

Aunque es un dispositivo muy necesario para dar asistencia a niños y adolescentes con una patología grave, casi es inexistente en todo el Estado. Solamente tienen este recurso las CCAA de Andalucía (son también Unidades de Salud Mental), Aragón (sólo para Trastornos de la Conducta Alimentaria), Canarias, Castilla la Mancha, Cataluña, Euskadi, Madrid y Navarra. En todas ellas, excepto en Cataluña y en Madrid, sólo hay un dispositivo.

- Dispositivos de Rehabilitación

- Unidades Hospitalarias de Rehabilitación/Comunidad terapéutica:

Es un dispositivo que se ha creado fundamentalmente en función del propio proceso de reforma de cada CA. Por ello, sólo en Andalucía y Asturias se denomina Comunidad Terapéutica. En el resto de las CCAA que cuentan con este dispositivo son Unidades/Clínica de Rehabilitación. En todos ellos se realiza una rehabilitación intensa durante un tiempo predeterminado.

- Centros de Rehabilitación Psicosocial

El análisis comparativo entre todas las CCAA ha sido dificultoso, comenzando por la definición del dispositivo que va desde Centro de Día, Programa, Unidad y/o Centro de Rehabilitación Psicosocial.

La disparidad de los Procesos de Reforma en cada CA desde su inicio en los años 80 , se refleja, sobre todo en esta prestación. La Ley General de Sanidad contempla la Rehabilitación Psicosocial como prestación sanitaria, sin embargo, en la mayor parte de las CCAA no dependen de las Consejerías de Sanidad sino de convenios o conciertos 
con Diputaciones, Cabildos, Ayuntamientos, Asociaciones de Familiares, Consejerías de Servicios Sociales, Conselleria de Bienestar Social, empresas públicas de gestión privada o privados. Exclusivamente es una prestación sanitaria en Andalucía, Asturias, Euskadi, Murcia y Rioja. En todos los dispositivos realizan Tratamientos rehabilitadores excepto en algún Centro de Día de Cataluña.

El número de plazas óptimas por 100.000 habitantes se sitúa en torno a 50 (Cuaderno Técnico $N^{0} 4$ de la AEN, 7 Pág. 59). Sin embargo ninguna CA llega a este número. Sólo Castilla la Mancha se le acerca con 47 plazas por 100.000 habitantes.

. Alternativas Laborales

La rehabilitación del enfermo mental es, en sí misma, un proceso de recuperación de las habilidades perdidas y de integración social y laboral. Dicho proceso incluye el uso de Talleres Ocupacionales, Talleres Protegidos, Centros de Rehabilitación Laboral o Empresas Sociales.

Si realizamos una valoración de los dispositivos existentes en las CCAA podemos observar que hay una gran carencia de los mismos en varias CCAA (no existe en Asturias, Melilla, Murcia, Rioja ni Valencia).

En las demás CCAA, según su Proceso de Reforma de la asistencia a la salud mental esta prestación se realiza de manera diferente. En algunas la ofrecen los Centros de Rehabilitación como última fase del Programa Individual de Rehabilitación (Canarias, Castilla la Mancha, Galicia y Madrid); en otras han sido las Asociaciones de Familiares quienes han asumido esta prestación (Andalucía: FAISEM). De Sanidad, sólo existen dos centros en Madrid que dependen administrativamente de la Consejería de Sanidad. En otras CCAA se presta mediante convenios con Diputaciones, Consell Insular, Servicios Sociales, Bienestar Social, Asociaciones de Familiares, ONGs, e incluso centros privados.

- Centros de Día

En este apartado se añadió la denominación de 0cio y Tiempo Libre. Sin embargo, la denominación de Centro de Día (Aragón, Asturias, Canarias y Cataluña) se utiliza para el dispositivo donde ser realizan las actividades de Rehabilitación Psicosocial y laboral.

Con este parámetro, queríamos saber y evaluar los dispositivos existentes en todas las CCAA donde se realizaran actividades de ocio y tiempo libre. De las respuestas, podemos concluir que su número es muy pequeño en todo el Estado y mayoritariamente son subvencionados por sanidad, aunque dependen administrativamente de Asociaciones de Familiares, Fundaciones, Cáritas, etc. En Euskadi dependen de la Comisión Sociosanitaria.

De la Consejería de Sanidad sólo dependen los de Cataluña, Aragón (uno), Galicia y Madrid. 
- Dispositivos Residenciales

. Alternativas Residenciales

Como hemos dicho anteriormente, en las distintas CCAA, según su propio proceso de transformación de la asistencia a salud mental, estos dispositivos se han ido creando conforme a las políticas sanitarias.

La estimación de plazas necesarias se calcula entre 65 y 130 (Cuaderno Técnico $\mathrm{n}^{0} 4$ de la AEN, Pág. 59, (7)).

En algunas CCAA se han implantado tres tipos de alternativas residenciales: pisos supervisados y/o tutelados, hostales y/o pensiones y residencias/hogares. En otras CCAA, programas residenciales (Aragón, Asturias y Madrid).

Respecto a la dependencia administrativa de los distintos dispositivos, es sanitaria exclusivamente en Asturias, Euskadi y Murcia. En Andalucía y Castilla la Mancha, dependen de Asociaciones y Fundaciones. En el resto de las CCAA, son concertados entre Sanidad, Cabildos, Diputaciones y Asociaciones de Familiares de Enfermos Mentales, centros privados, ONGs, etc.

. Unidades de Media Estancia

Solamente no existe este dispositivo en Andalucía y en Melilla. Los datos aportan información de que este dispositivo, en el resto de las CCAA, es sobre todo residencial y suele ubicarse en las dependencias de los antiguos Hospitales Psiquiátricos. Excepto en Navarra, Rioja y Valencia se realiza algún tipo de Tratamiento rehabilitador.

. Unidades de Larga Estancia

Las CCAA de Andalucía, Asturias, Cantabria, Melilla y Navarra no tienen ningún dispositivo de este tipo.

En el resto de CCAA son, sobre todo dispositivos residenciales ubicados en las dependencias de los antiguos Hospitales Psiquiátricos. Se realizan cuidados específicos, aunque en algún dispositivo también prestan programas rehabilitadores.

Su dependencia es casi siempre pública, bien de sanidad o diputaciones y / consorcios sanitarios o empresas públicas. En la CCAA de Murcia la dependencia administrativa es del Instituto Murciano de Servicios Sociales.

\section{. Psicogerátricos}

A pesar de que en la Ley General de Sanidad se especificó la necesidad de ofrecer esta prestación, en la mayoría de las CCAA no la ofrecen y, en algunas es una prestación social, no sanitaria (Extremadura, Madrid y Murcia). Es significativo que en Cantabria todos los dispositivos sean privados.

\section{Profesionales}

No hemos encontrado en ningún documento cual sería el porcentaje de Profesionales necesarios para ofrecer una atención a la salud mental de calidad en todos los dispositivos de la Red de Salud Mental. Las únicas estimaciones de profesionales que hemos encontrado (Cuaderno Técnico $n^{0} 4$ de la AEN, 7) ya han sido presentadas. 
Como hemos podido observar en los datos presentados, si realizamos el análisis comparativo entre las distintas CCAA en cada Profesión y según el Total de Profesionales, la dispersión es muy demostrativa de la situación de cada CCAA.

Según las profesiones:

- Psiquiatras: La media de todo el Estado está en torno a $6(6,52)$.

- Psicólogos: La media se sitúa en torno a $4(4,28)$.

- Enfermería: La media está en torno al $9(9,4)$.

- Trabajador Social: La media se acerca al $2(1,88)$.

- Terapeuta 0cupacional: La media de las CCAA (excepto Cataluña y Valencia) está en 0,78

- Auxiliares de Clínica / Monitores: No tenemos datos de tres CCAA (Cantabria, Cataluña ni Murcia). Del resto de CCAA, la media está en torno al $15(15,03)$.

- Otros: No tenemos datos de Aragón, Cantabria, Cataluña ni la Rioja. La media del resto de CCAA está en torno a $11(11,02)$.

En los datos, es demostrativa la incorporación progresiva de nuevas profesiones en todos los dispositivos de atención a la salud mental. Sobre todo se han ido incorporando Terapeutas Ocupacionales en las unidades de Hospitalización Breve tanto de adultos como infantil. También ha ido aumentando significativamente el número de Trabajadores Sociales y Monitores (de Rehabilitación Psicosocial).

Respecto al análisis comparativo del Total de Profesionales en la Red asistencial de salud mental entre las distintas CCAA, podemos decir en resumen que los datos muestran que Euskadi es la que cuenta con el mayor número, siendo Murcia la que cuenta con menos profesionales tiene (recordamos que carecemos del número de Auxiliares de Clínica). 